\title{
Foreign Students' Self-Confidence in Learning Chinese
}

\author{
Aidin Tajbakhsh, Khairuddin Khairkhah \\ Central China Normal University, Wuhan, Hubei, China
}

\begin{abstract}
This survey study was aimed to inspect different amount of self-confidence experienced by foreign students in learning Chinese. Forty-two students from the CICE (College of International Cultural Exchange) of CCNU (Central China Normal University), in Wuhan, in spring 2013, has been randomly given a questionnaire about their motivation, effort, self-confidence, and the subjective evaluation of the effect of their own native languages on their overall performance in Chinese. It was assumed that phonological similarity between learners' native language and Chinese can enhance their self-confidence, and consequently, increase their performance. However, since processing an actual CLS (cross-language similarity) is beyond the scope of this study, and the perceptual similarity evaluation of individuals has shown to be inaccurate, the study has failed to find such a relationship, and even found some negative links. Nevertheless, result shows a significant effect of the number of languages participant speak on their perceived performance. Also their perceived performance has a significantly positive correlation with some self-confidence components.
\end{abstract}

Keywords: learning chinese, self-confidence, phonological similarity, perceived language similarity, PAM (Perceptual Assimilation Model), SLM (Speech Learning Model)

\section{Introduction}

Individuals vastly vary on their performance level in L2 (the second language) learning. Both cognitive and affective aspects of individuals affect the process of language learning (Park \& Lee, 2005). Some of these variations can be attributed to the personal, or intrinsic, factors, such as self-confidence. Self-confidence is a positive belief that people have about their ability to overcome a specific situation; facing challenges successfully and mastery experiences can increases one's self-confidence, which, in turn, increases the likelihood of individuals' engaging in related behavior and enhancing their performance (D. H. Hockenbury \& S. E. Hockenbury, 2006). Several studies (e.g., Heyde, 1979, as cited in Park \& Lee, 2005) have shown the positive correlation between self-confidence and oral performance of L2 learners (Park \& Lee, 2005). Self-confidence, or interchangeably self-esteem, consist of three global, situational, and task levels; an example of task self-esteem, which is the self-evaluation one makes in particular tasks of specific situations, is one's own assessment in a particular language skill such as listening, speaking, reading, and writing (Park \& Lee, 2005).

On the other hand, extrinsic factors, which are resulted by the experience of L2 learning itself, can also affect both cognitive and affective aspects of a L2 learner (Kanafani, 2009). One such a factor that has been known to have an effect on many aspects of language learning, such as (L2) perception and production is

Aidin Tajbakhsh, Master's Degree, Central China Normal University. Khairuddin Khairkhah, Master's Degree, Central China Normal University. 
phonological similarity, that is, similarity between two sound structures (segments, syllables, prosodic patterns, etc.) of, for example, L1 and L2 (Chang, n. d.). This sound-based similarity can further be divided into about five subtypes, namely objective acoustic similarity, syntagmatic phonetic similarity, paradigmatic phonemic similarity, articulatory/gestural similarity, and aerodynamic similarity (Chang, n. d.). Acoustic similarity, which is sounds distance in acoustic aspects such as frequency, duration, and amplitude, is not specific to any language, and phonetic similarity, which refers to how related two sounds are to each other within a language, is specific to one language, whereas phonemic similarity is a cross-language comparison between sounds at the level of context-free phonemes in two aspects of: (1) similarity in positions of "relative phonetics" in the respective phonemic inventories; and (2) similarity of their distribution in the two languages (Chang, n. d.).

Several linguistic theories account for the effect of phonological similarity over multiple aspects of language learning. One such a theory is the PAM (Perceptual Assimilation Model), according to which, the phonological structure of L1 greatlty effects the speech perception of another language, which means that listeners of different language backgrounds would interpret the same sound in L2 differently.

According to another such a theory, which is the SLM (Speech Learning Model), L2 learners classifies L2 sounds, in comparison to familiar L1 sounds, as either "new", "similar", or "identical". An "identical" L2 sound has a phonetically the same counterpart in L1, while a "similar" L2 sound can be significantly different from its L1 counterpart phonetically. In contrast, a "new" L2 sound has practically no clear L1 counterpart. It is suggested by this model that identifying an L2 sound as either "identical", "similar", or "new" can profoundly affect its production (Chang, n. d.).

Thus, the next logical question that arises is whether there is any inter-mediatory effect of phonological similarity over the L2 learners' self-confidence. Another question that comes up is that in which direction and what magnitude this effect can be. In our literature review, however, we were unable to discover such an inter-disciplinary study, especially, the one that address the way that confronting a phonologically complex, and novel, language system, like Chinese, can affect CLL (Chinese language learners') self-confidence in learning further Chinese; and we thought that the implication of finding such a relationship would be the need to adjust the current teaching methods for foreigners, in order to boost their self-confidence, by emphasizing more on the phonological aspects of Chinese language, particularly at the basic level.

Therefore, we aimed in this study to look for a possible relationship between CLLs' cross-language similarity and their self-confidence, and the way that these two together may influence the CLLs' overall performance. We speculated that the phonological similarity of learners' own spoken language(s) to Chinese could have a mediatory effect in increasing their self-confidence, and in turn, would enhance their performance in learning Chinese.

\section{Method}

\section{Participants}

From a population of foreign students at the CICE (College of International Cultural Exchange) of CCNU (Central China Normal University), in Wuhan, we selected our sample by randomly approaching foreign individuals, either sitting, or passing by, at an area outside the CICE dormitory buildings 5 and 6 . Forty-two foreign students from 31 different countries, and with an age range of 17 to 36 ( $M=23.31 \mathrm{yrs}$ ), out of which 27 were males and 15 were females, have participated in this study, with about $19 \%$ non-response rate. 


\section{Procedure}

During the spring semester of 2013, after explaining the purpose of our study, the questionnaire consisting of 19 question items were given to those foreign students at CICE who consented to participate. Five of those items were demographic data, and other seven were background information such as motivation level, and effort. The focal items were designed to measure factors such as students' attitude toward Chinese language skills, some aspect of self-confidence, and their cross-language similarity level. Several phonetic similarity measurement methods have been developed so far to make such a comparison across languages (e.g., Htun, Kodama, \& Mikami, 2011, using the international phonetic alphabet and phonetic matching techniques). However, since processing an actual CLS (cross-language similarity) is beyond the scope of this study, we have decided to use individuals' PLS (perceived language similarity), and the number of languages they know, assuming that any new language could have had increased their phonetic repertoire, and therefore increasing the likelihood of perceiving Chinese sounds as more familiar.

Before conducting the main survey, a draft version of the questionnaire was administered to some volunteer colleagues to check for the face validity of the items. Throughout the actual trial, the investigators have tried to clarify any ambiguity, should participants request.

\section{Results}

First, using the focal items, after omitting two problematic items (Q15, Q16b), which both also have 9.5\% missing rate, the scale shows a quite moderate reliability $(\alpha=0.59)$. The data, however, has failed to produce an acceptable construct validity, when the focal items' (Q13-Q17) total score was correlated with the general self-rating item (Q18) $(r=0.146, p=0.387)$.

Using factor analysis on this scale, with eigenvalue greater than one, we were unable to produce any distinctive factor. Nonetheless, borrowing from other studies on the effect of self-confidence on L2 learning (i.e., Park \& Lee, 2005), we have tried to dub some of our variable items on self-confidence (i.e., Q16, Q17), that were closely resembling their factor items, as subcategories such as $\mathrm{C}-\mathrm{C}$ (communication confidence), and P-C (potential confidence) (respectively), for the sake of easing their comprehension. Furthermore, the number of means students used to study Chinese (Q11) was added to the number of hours they spent studying (Q12), to form an effort variable; Q13a to Q13d, which measured respondents' feeling about Chinese language skills, were added up and dubbed attitude; Q14 was titled P_P (perceived performance); and Q17a to Q17d were summed up as their PLS factor. Table 1 is the correlation among this study's main variables:

According to this data, students' self-assessment is positively correlated with their potential confidence $(r$ $=0.50, p<0.001)$ and the amount of effort they put forth to study Chinese $(r=0.40, p=0.004)$; also there are positive relations between participants' attitude on Chinese language skills and their $\mathrm{P}_{-} \mathrm{C}(r=0.47, p=0.001)$, and effort $(r=0.33, p=0.017)$, which is in line with the common sense, and previous findings.

Moreover, the number of known Languages produced a significantly positive correlation with $\mathrm{P} \_\mathrm{P}(r=$ $0.42, p=0.004)$, which supports the hypothesis. However, this variable, and assessment on own-language similarity to Chinese, has both created strongly negative relations with C_C $(r=0.33, p=0.026$, and $r=0.25, p$ $=0.068$, correspondingly), and generally, these two factors are found to have been negatively related with other main variables of the scale.

Based on this observation a model has been designed (see Figure 1), and two multi-linear regression procedures have been devised, to test for the study's hypothesis. 
Table 1

Correlations

\begin{tabular}{|c|c|c|c|c|c|c|c|c|}
\hline & & Effort & Attitude & $\mathrm{C} \_\mathrm{C}$ & P_C & PLS & P_P & Languages \\
\hline \multirow{2}{*}{ Effort } & Pearson Correlation & 1 & $0.331^{*}$ & 0.033 & $0.396^{* *}$ & -0.067 & $0.404^{* *}$ & 0.137 \\
\hline & Sig. (1-tailed) & & 0.017 & 0.423 & 0.005 & 0.338 & 0.004 & 0.203 \\
\hline \multirow{2}{*}{ Attitude } & Pearson Correlation & & 1 & -0.025 & $0.471^{* *}$ & 0.112 & 0.219 & 0.081 \\
\hline & Sig. (1-tailed) & & & 0.442 & 0.001 & 0.246 & 0.085 & 0.312 \\
\hline \multirow{2}{*}{ C_C } & Pearson Correlation & & & 1 & 0.190 & -0.250 & -0.012 & $-0.332^{*}$ \\
\hline & Sig. (1-tailed) & & & & 0.130 & 0.068 & 0.471 & 0.026 \\
\hline \multirow{2}{*}{$\mathrm{P}_{-} \mathrm{C}$} & Pearson Correlation & & & & 1 & -0.028 & $0.501^{* *}$ & -0.050 \\
\hline & Sig. (1-tailed) & & & & & 0.431 & 0.000 & 0.381 \\
\hline \multirow{2}{*}{ PLS } & Pearson Correlation & & & & & 1 & -0.178 & -0.044 \\
\hline & Sig. (1-tailed) & & & & & & 0.133 & 0.397 \\
\hline \multirow{2}{*}{ P_P } & Pearson Correlation & & & & & & 1 & $0.419^{* *}$ \\
\hline & Sig. (1-tailed) & & & & & & & 0.004 \\
\hline \multirow{2}{*}{ Languages } & Pearson Correlation & & & & & & & 1 \\
\hline & Sig. (1-tailed) & & & & & & & \\
\hline
\end{tabular}

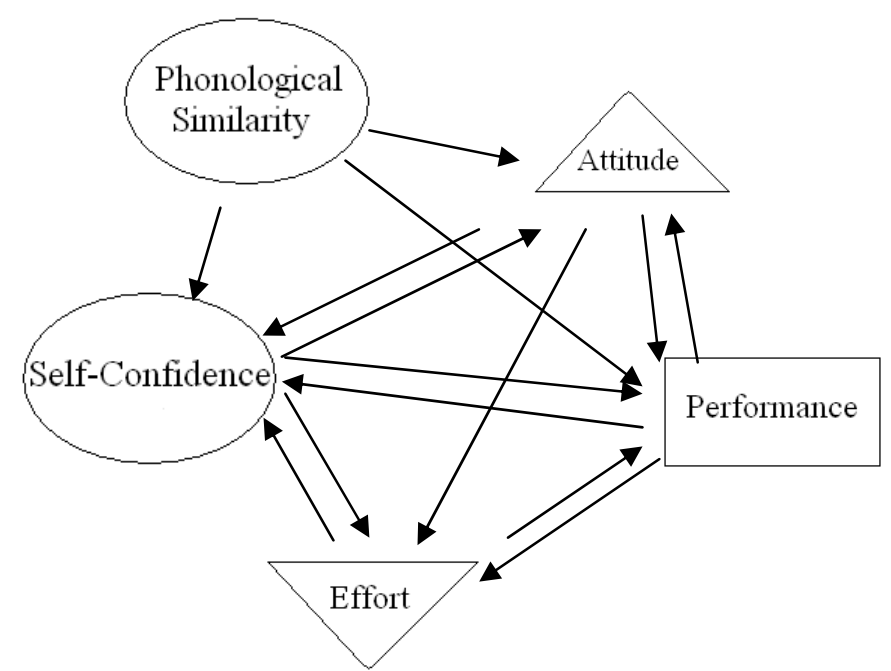

Figure 1. The hypothetical model.

Reg1: $P_{-} P=b_{0}+b_{1}$ effort $+b_{2}$ attitude $+b_{3}$ self_confidence $+b_{4}$ phonological_similarity

This model has failed to produce a significant relation between predictors and the outcome (P_P).

Reg2: self_confidence $=b_{0}+b_{1}$ effort $+b_{2}$ attitude $+b_{3} P \_P+b_{4}$ phonological_similarity

This second model could find a significant predictability for the self-confidence from some of the variables. However, here the PLS shows a slightly negative direction with the outcome.

One interesting finding of our analysis, though, was that on average females reported a significantly higher amount of effort $(M=4.73, S D=1.53, S E=0.40), t(40)=-2.247, p=0.030$, more positive attitude $(M=12.53, S D=1.68, S E=0.43), t(39)=-3.028, p=0.004$, and more optimistic expectation for their achievement $(M=4.93, S D=0.96, S E=0.25), t(40)=-2.383, p=0.022$, than those of men for effort $(M=3.67, S D=1.44, S E=0.28)$, attitude $(M=10.35, S D=2.48, S E=0.49)$, and expectation $(M=3.81, S D$ $=1.66, S E=0.32)$. 
Table 2

Model Summary

\begin{tabular}{lllll}
\hline & & Model summary & \\
\hline Model & $R$ & $R$-square & Adjusted $R$-square & Std. error of the estimate \\
\hline 1 & $0.606^{\text {a }}$ & 0.368 & 0.295 & 1.24946 \\
\hline
\end{tabular}

Note. a. Predictors: (Constant), PLS, effort, P_P, attitude.

Table 3

ANOVA $^{\mathrm{b}}$

\begin{tabular}{lllrlll}
\hline \multicolumn{7}{c}{ ANOVA $^{\mathrm{b}}$} \\
\hline Model & & Sum of squares & $d f$ & Mean square & $F$ & Sig. \\
\hline \multirow{3}{*}{1} & Regression & 31.760 & 4 & 7.940 & 5.086 & $0.002^{\mathrm{a}}$ \\
& Residual & 54.640 & 35 & 1.561 & & \\
& Total & 86.400 & 39 & & & \\
\hline
\end{tabular}

Notes. a. Predictors: (Constant), PLS, effort, P_P, attitude; b. Dependent Variable: P_C.

Table 4

Coefficients $^{\mathrm{a}}$

\begin{tabular}{|c|c|c|c|c|c|c|}
\hline \multicolumn{7}{|c|}{ Coefficients $^{\mathrm{a}}$} \\
\hline \multirow{2}{*}{\multicolumn{2}{|c|}{ Model }} & \multirow{2}{*}{$\begin{array}{l}\text { Unstandardized coefficients } \\
B\end{array}$} & \multicolumn{2}{|r|}{ Standardized coefficients } & & \multirow{2}{*}{ Sig. } \\
\hline & & & Std. Error & Beta & & \\
\hline \multirow{5}{*}{1} & (Constant) & -0.299 & 1.236 & & -0.242 & 0.810 \\
\hline & Effort & 0.288 & 0.137 & 0.305 & 2.108 & 0.042 \\
\hline & Attitude & 0.198 & 0.089 & 0.328 & 2.233 & 0.032 \\
\hline & P_P & 0.294 & 0.191 & 0.214 & 1.538 & 0.133 \\
\hline & PLS & -0.012 & 0.065 & -0.025 & -0.180 & 0.859 \\
\hline
\end{tabular}

Note. a. Dependent Variable: P_C.

\section{Discussion}

Individuals' variation in L2 performance, which in this study was Chinese language, can be attributed to both cognitive and affective factors, among which are personality characteristics, such as self-confidence (Park \& Lee, 2005). Also phonological similarity of L2 to L1 has been shown to make a great contribution to L2 performing quality, as it is explained by theories such as PAM, and SLM (Chang, n. d.). Thus, to find whether there is an inter-relation between phonological similarities of students owns language(s) to Chinese and their self-confidence, we conducted this survey study, hypothesizing that the more similar their languages are to Chinese, the higher would be their self-confidence.

The current results have failed to support this hypothesis, which can be due to several limitations of this survey, including a weak construction of the scale and the use of problematic variables, the first of which was individuals' PLS assessment. As it is explained by Chang (n. d.), a pair of sounds that are acoustically and/or phonetically similar in two languages may nonetheless be phonemically dissimilar, and vice versa. However, unlike similarity perception within a language, an accurate similarity assessment between two languages requires a high level of expertise on the second language. Therefore, due to the fact that most participants lack an adequate knowledge of the Chinese language, the PLS of individuals is inaccurate and does not account for the objective phonological similarity across languages. 
Another limitation of this study was, generally, a small number of participants, and particularly, the underrepresentation of each foreign language represented in the sample data. Moreover, the high incorrect responses of some individuals, and the high missing rate of some of the items, might indicate the ambiguity in those items, which could be also due to limited English ability, which was the medium of this scale.

Yet another major limitation of our study was the linear nature of our assumption. As explained by the PAM, the difficulty in discriminating a foreign sound arises as the individuals gain more experience with their L1, also acquiring other languages, which leads to a higher likelihood of a listener's native phonology interfering the L2 production as a deviant sound from L2 sounds perceptually assimilated to the same native sound (Chang, n. d.). Furthermore, according to SLM, while L2 learners should not have difficulties in producing an "identical" L2 sound, as its differences to the respective L1 sound is negligible, and in articulating a "new" L2 sound, since it does not have any L1 correspondence, their speech production should be negatively affected when they identify an L2 sound as "similar" to an L1category because of the significant phonetic disparity between the L1 and L2 sounds (Chang, n. d.). Hence, perhaps, instead of a linear relationship between the learners self-confidence and the quality of L2 (i.e., Chinese acquisition in this study), mediated by the level of similarity between two languages, as we have hypothesized, the two variables might have a quadratic correlation, with the highest self-confidence, and the best performance, when L1 and L2 are the least and the most phonologically similar, and a decreased one as the two languages' similarity depart from the extremes.

Thus it is up to future studies, using the international phonetic alphabet and phonetic matching techniques, to measure the objective phonetic similarity across language with the more representative samples, to find whether it has a mediatory effect on L2 learners' self-confidence.

\section{References}

Chang, Ch. B. (n. d.). Determining cross-linguistic phonological similarity between segments: The primacy of abstract aspects of similarity. In Cairns, C., \& Raimy, W. E. (Eds.), The segment in phonology and phonetics. Retrieved at April 20, 2013, from http://linguistics.berkeley.edu/ cbchang/papers/Chang_SegmentVol.pdf

Hockenbury, D. H., \& Hockenbury, S. E. (2006). Psychology (4th edition). N.Y., USA: WORTH Publishing.

Htun, O., Kodama, Sh., \& Mikami, Y. (2011). Cross-language phonetic similarity measure on terms appeared in asian languages. International Journal of Intelligent Information Processing, 2(2), 9-21. Retrieved from http://www.state.gov/misc/list/

Kanafani, J. K. (2010). The importance of building students' self-esteem in the language learning. المجله التربويه 34), 44), 31). Retrieved at April 24, 2013, from http://www.crdp.org/crdp/Arabic/ar-news/majalla_ar/pdf44/44_P31_34.pdf

Park, H., \& Lee, R. A. (2005). L2 learners' anxiety, self-confidence and oral performance. The 10th Pan-Pacific Association of Applied Linguistics (PAAL) Japan Proceedings. Retrieved at April 24, 2013, from http://www.paaljapan.org/resources/ proceedings/PAAL10/pdfs/hyesook.pdf

\section{Appendix}

\section{Background items}

6. How long have you been in china?
a) Less than 6 months
b) 6 months to 1 year
c) 1 to 2 years
d) More than 2 years

7. What was your main motive to come to china? (choose all that apply)
a) To learn Chinese
b) To live abroad
c) It was financially the best choice to study
d) My study major

was related to Chinese culture/history/politic/etc

8. If you choose item (a) on Q7 please answer this question, otherwise go to Q9. What was your main motive to choose to learn Chinese? 

a) I find Chinese interesting
b) It is an important language for the business/work
c) It is important to learn more about Chinese culture d) Other

9. What was your Chinese level before coming to china?
a) Not at all
b) Few words and phrases
c) Basic conversation
d) Intermediate-Advance

10. Now that you are in china do you feel it is necessary to study (further) Chinese?

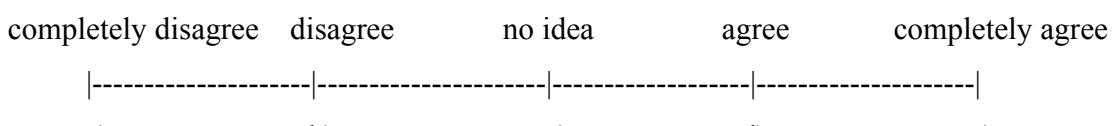

a)

b)

c)

d)

e)

11. What mean(s) do/did you use to study Chinese? (choose all that apply)
a) Self-study
b) Chinese friends
c) Private mentor
d) Public/regular Chinese class

12. On average, how many hours per day do/did you study Chinese?
a) Less than 1 hour
b) 1 to 2 hours
c) 3 to 4 hours
d) More than 4 hours

\section{Focal items}

13. How do you feel about the following language skills in Chinese?

\begin{tabular}{|c|c|c|c|c|c|}
\hline & very hard & hard & fair & easy & very easy \\
\hline Reading & a) & b) & c) & d) & e) \\
\hline Writing & a) & b) & c) & d) & e) \\
\hline Speaking & a) & b) & c) & d) & e) \\
\hline Listening & a) & b) & c) & d) & e) \\
\hline
\end{tabular}

14. How do you rate your performance in learning Chinese compare with other students (in your level/ class)? bottom Pretty bad slightly bad average slightly good pretty good top

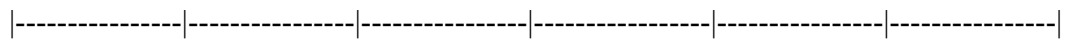
a)
b)
c)
d)
e)
f)
g)

If you have ever learned any foreign language before (other than Chinese)-including English- please answer this question, otherwise go to Q16.

15. Compare to other language class(es) that you take/took, how do you do in the Chinese class?

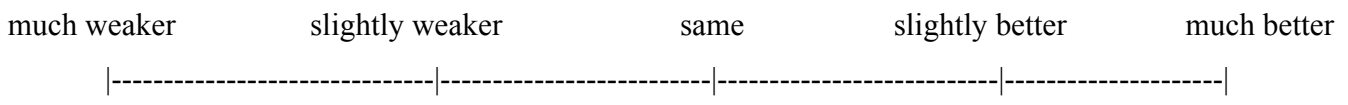
a)
b)
c)
d)
e)

16. When you should talk to strangers in Chinese, how do you describe yourself?

\begin{tabular}{|c|c|c|c|c|c|}
\hline comp & tely disagree & disagree & no idea & agree & completely agree \\
\hline & & ------- & --"----- & --|----- & -------| | \\
\hline Shy & a) & b) & c) & d) & e) \\
\hline Confident & a) & b) & c) & d) & e) \\
\hline Afraid of making & a) & b) & c) & d) & e) \\
\hline
\end{tabular}

mistakes

17. How similar or different Chinese language is in each of the following aspects to your own native language(s)?

$$
\text { very different } \quad \text { slightly diff. no idea slightly similar very similar }
$$

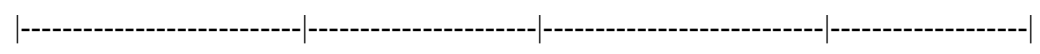


Vowels

$(\bar{a}, \breve{a}, \grave{a}, e, i, o, \ddot{o}, \ldots)$

Characters'

a)

sound

Grammatical

a)

structure

Body language b)

b)

b)

b) c)

d)

d)

d)

c)

c) e)

e)

18. Overall, how much do you think your own language(s) influence your learning Chinese?

big disadvantage slightly disadvantageous no effect slightly advantageous

big advantage

b)

c)

d)

e)

19. Comparing to the new HSK Chinese proficiency exam, what level of language mastery are you expecting to achieve by the end of your study in china?

a. Level I (to understand and use very simple Chinese words and phrases.)

b. Level II (to communicate easily in simple and routine tasks on familiar and routine matters.)

c. Level III (to communicate in Chinese at a basic level in daily, academic and professional lives.)

d. Level IV (to communicate fluently with native Chinese speakers on a wide range of topics.)

e. Level V (to comprehend Chinese newspapers, films, and give a full-length speech in Chinese.)

f. Level VI (to fully comprehend/express written and spoken Chinese.) 